POS $\quad$ PROCEEDINGS

\title{
Commissioning of Belle II data acquisition
}

\author{
SeokHee Park ${ }^{* \dagger}$ \\ Yonsei University, Seoul, Korea \\ E-mail: seokhee.park@yonsei.ac.kr
}

Mikihiko Nakao, Ryosuke Itoh, Satory Yamada, Soh Suzuki

High Energy Accelerator Research Organization, Tsukuba, Japan

E-mail: mikihiko.nakao@kek.jp

\section{Tomoyuki Konno}

Kitasato University, Tokyo, Japan

E-mail: tkonnoekitasato-u.ac.jp

\section{Youngjoon Kwon}

Yonsei University, Seoul, Korea

E-mail: yjkwon63@yonsei.ac.kr

The Belle II experiment operates at the SuperKEKB $e^{+} e^{-}$energy-asymmetric collider on or near the $\Upsilon(4 S)$ resonance energy. The Belle II experiment has completed the "Phase 2" run, in which the Belle II operation without the vertex detector system has been tested. The main focus of the Phase 2 run is on the search for new physics related with dark sector, axion-like particles, etc. with an intermediate luminosity on the way to reach the challenging target. To accomplish these physics goals of Phase 2, a new trigger menu for the single photon and low multiplicity events is essential. With the luminosity design goal, it is crucial to cope with a high trigger rate for data acquisition (DAQ). In this presentation, we describe the DAQ system of Belle II, focusing on the successful commissioning in Phase 2 and the performance of the system.

ICHEP 2018, International Conference of High Energy Physics

4-11 July 2018

Seoul, Korea

\footnotetext{
*Speaker.

${ }^{\dagger}$ on behalf of the Belle II DAQ group
} 


\section{Overview}

The Belle II experiment[1] is designed to search for new physics beyond the Standard Model[2] using high-statistics sample of $B$, charm, and $\tau$ which are generated by SuperKEKB[3] $e^{+} e^{-}$collider. The target luminosity of SuperKEKB is $8 \times 10^{35} \mathrm{~cm}^{-2} \mathrm{~s}^{-1}$, and integrated luminosity goal is $50 \mathrm{ab}^{-1}$. Accordingly, the DAQ system is designed[4, 5] to cope with the high luminosity data which is taken from 7 sub-detector components. DAQ read out all detector signals upon L1 trigger and build event in multiple steps [6] with High Level Trigger (HLT)[7].

One of the important concept of the Belle II DAQ is unification. We unify the firmware interface in frontend electronics (FEE) boards for trigger-timing distribution (TTD/FTSW)[8] and data flow (Belle2link)[9]. Data flow from FEE to HLT is also unified. This unification allows to reduce costs and resources for managing and maintaining system.

\section{Phase 2 Result and Phase 3 Plan}

From April to July, 2018, Belle II phase 2 data taking was performed. During phase 2 run, our DAQ was successfully commissioned as designed. First, full event process chain was working well. Second, capability to handle up to $30 \mathrm{kHz}$ with dummy trigger and limited sub-detector systems was confirmed. In actual data taking, L1 trigger rate was kept up to $500 \mathrm{~Hz}$ due to phase 2 beam, background status and trigger condition.

For phase 3 full data taking, we need to include full PXD and SVD readout system and prepare frontend firmware for $30 \mathrm{kHz}$ L1 trigger rate. Also, more stable DAQ operation is required. For the stability, comprehensive error reporting system and faster, more automated recovery procedures will be prepared. These reduce downtime and deadtime fraction.

\section{References}

[1] Belle-II collaboration, T. Abe et al., Belle II Technical Design Report, 1011.0352.

[2] Belle II collaboration, E. Kou et al., The Belle II Physics Book, 1808.10567.

[3] K. Akai, K. Furukawa and H. Koiso, SuperKEKB collider, Nucl. Instrum. Methods Phys. Res. A 907 (2018) 188 .

[4] M. Nakao, T. Higuchi, R. Itoh and S. Y. Suzuki, Data acquisition system for Belle II, Journal of Instrumentation 5 (2010) C12004.

[5] S. Yamada, R. Itoh, K. Nakamura, M. Nakao, S. Y. Suzuki, T. Konno et al., Data Acquisition System for the Belle II Experiment, IEEE Transactions on Nuclear Science 62 (2015) 1175.

[6] S. Y. Suzuki, S. Yamada, R. Itoh, M. Nakao, T. Konno, T. Higuchi et al., The Three-Level Event Building System for the Belle II Experiment, IEEE Transactions on Nuclear Science 62 (2015) 1162.

[7] R. Itoh, T. Higuchi, M. Nakao, S. Y. Suzuki and S. Lee, Data Flow and High Level Trigger of Belle II DAQ System, IEEE Transactions on Nuclear Science 60 (2013) 3720.

[8] M. Nakao, Timing distribution for the Belle II data acquistion system, Journal of Instrumentation 7 (2012) C01028.

[9] D. Sun, Z. Liua, J. Zhao and H. Xu, Belle2Link: A Global Data Readout and Transmission for Belle II Experiment at KEK, Physics Procedia 37 (2012) 1933. 